Draft Version November 24, 2018

Preprint typeset using $\mathrm{IAT}_{\mathrm{E}} \mathrm{X}$ style emulateapj v. 12/16/11

\title{
DISCOVERY OF A COMPACT COMPANION TO THE HOT SUBDWARF STAR BD $+37^{\circ} 442$
}

\author{
Nicola la Palombara, Sandro Mereghetti \\ INAF - IASF Milano, via Bassini 15, I-20133 Milano, Italy \\ Andrea TiEngo \\ IUSS-Istituto Universitario di Studi Superiori, viale Lungo Ticino Sforza 56, I-27100 Pavia, Italy \\ PAOLO Esposito \\ INAF, Osservatorio Astronomico di Cagliari, località Poggio dei Pini, strada 54, I-09012, Capoterra, Italy \\ Draft version November 24, 2018
}

\begin{abstract}
We report the results of the first $\mathrm{X}$-ray observation of the luminous and helium-rich O-type subdwarf $\mathrm{BD}+37^{\circ} 442$ carried out with the $X M M-$ Newton satellite in August 2011. X-ray emission is detected with a flux of about $3 \times 10^{-14} \mathrm{erg} \mathrm{cm}^{-2} \mathrm{~s}^{-1}(0.2-1 \mathrm{keV})$ and a very soft spectrum, well fit by the sum of a blackbody with temperature $k T_{\mathrm{BB}}=45_{-9}^{+11} \mathrm{eV}$, and a power law with a poorly constrained photon index. Significant pulsations with a period of $19.2 \mathrm{~s}$ are detected, indicating that the $\mathrm{X}$-ray emission originates in a white dwarf or neutron star companion, most likely powered by accretion from the wind of $\mathrm{BD}+37^{\circ} 442$.
\end{abstract}

Subject headings: Stars: individual: $\mathrm{BD}+37^{\circ} 442$ - subdwarfs — pulsars: general — X-rays: stars — $\mathrm{X}$-rays: binaries

\section{INTRODUCTION}

Hot subdwarfs are evolved, low-mass stars that have lost most of their hydrogen envelope and are now in the stage of helium-core burning (see Heber 2009, for a review). From the spectroscopic point of view we distinguish the cooler B-type subdwarf (sdB) stars, with effective temperature $T_{\text {eff }}<40,000 \mathrm{~K}$, and the hotter O-type (sdO) stars, with $T_{\text {eff }}>40,000 \mathrm{~K}$ (Hirsch et al. 2008). Most sdB stars are helium poor and display no or only weak helium lines, while most sdO stars are helium rich and show He I and He II lines. While the sdBs form a homogenous group, the sdOs show a wide spread in temperature $\left(T_{\text {eff }}=40,000-100,000 \mathrm{~K}\right)$, gravity $(\log (g)$ $=4-6.5)$ and helium abundance (Heber \& Jefferv 1992; Heber et al. 2006). Historically, sdO stars were divided into 'luminous' and 'compact' sdOs, depending on their value of $\log (g)$.

One possible mechanism responsible for the loss of the massive hydrogen envelopes of hot subdwarfs is mass transfer in a binary. Indeed, there is substantial evidence that many hot subdwarfs are in close binary systems (Maxted et al. 2001; Napiwotzki et al. 2004; Copperwheat et al. 2011). In particular, a large fraction of binaries is found among sdB stars. Models of binary evolution (Han et al. 2002) predict that many of the subdwarf companions should be white dwarfs (WD). Indeed a few sdB+WD are known (Koen et al. 1998; Maxted et al. 2000; Edelmann et al. 2005), and recent optical studies of single-lined spectroscopic binaries yielded several new candidates (Geier et al. 2010). The presence of a compact companion (a WD, neutron star (NS), or black hole) could be revealed by the detection

\footnotetext{
nicola@iasf-milano.inaf.it, sandro@iasf-milano.inaf.it andrea.tiengo@iusspavia.it paoloesp@oa-cagliari.inat.it
}

of X-ray emission powered by accretion, if the subdwarf mass-donor can provide a sufficiently high accretion rate. Short Swift/XRT observations of candidate $\mathrm{sdB}+\mathrm{WD} / \mathrm{NS}$ binaries, selected from the Geier et al. (2010) sample, gave X-ray luminosity upper limits of $\sim 10^{30}-10^{31} \mathrm{erg} \mathrm{s}^{-1}$ (Mereghetti et al. 2011a). These limits confirm that sdB stars have rather weak stellar winds, unable to provide enough accretion rate.

Although the fraction of binaries among sdO stars is not as high as for the sdBs, the prospects to find $\mathrm{X}$ ray emitting companions are more promising for these stars. In fact, at least a few luminous sdO stars show evidence for stellar winds with mass loss rate $\dot{M} \sim$ $10^{-7}-10^{-10} M_{\odot} \mathrm{yr}^{-1}$ (Jeffery \& Hamann 2010). Among these, the bright star HD 49798 is known as a soft Xray source, with a strong periodic modulation at $13.2 \mathrm{~s}$, since more than 15 years (Israel et al. 1997). More recent $\mathrm{X}$-ray observations with $X M M-N e w t o n$ showed that the compact companion of HD 49798 is a massive (1.28 \pm $\left.0.05 M_{\odot}\right)$ white dwarf (Mereghetti et al. 2009). To our knowledge, no other $\mathrm{X}$-ray detections of sdO stars have been reported up to now.

Prompted by our findings on HD 49798, we carried out an X-ray observation of another bright sdO star. Our target is $\mathrm{BD}+37^{\circ} 442$ (Rebeirot 1966), a luminous sdO which is often referred to as an 'extreme helium star' because of its complete lack of hydrogen (while HD 49798 has about $20 \%$ (by mass) of hydrogen): it shows evidence for significant mass loss rate (Jeffery \& Hamann 2010) and stellar parameters similar to those of HD 49798 (see Tab. 1), but with the notable difference that, up to now, no evidence of binary nature was reported. Here we present the results of the first X-ray observations of $\mathrm{BD}+37^{\circ} 442$, which indicate the presence of a compact companion, either a WD or a NS. 
TABLE 1

MAIN PARAMETERS OF THE SDO STARS BD +37 442 AND HD 49798

\begin{tabular}{|c|c|c|c|c|c|}
\hline \multirow[t]{2}{*}{ Parameter } & \multirow[t]{2}{*}{ Symbol } & \multicolumn{2}{|c|}{$\mathrm{BD}+37^{\circ} 442$} & \multicolumn{2}{|c|}{ HD 49798} \\
\hline & & Value & Reference & Value & Reference \\
\hline $\operatorname{Mass}\left(M_{\odot}\right)$ & $\bar{M}$ & 0.9 & 1 & $1.50 \pm 0.05$ & 5 \\
\hline $\operatorname{Radius}\left(R_{\odot}\right)$ & $R$ & 1.6 & 1 & $1.45 \pm 0.25$ & 6 \\
\hline Surface gravity & $\log g$ & $4.0 \pm 0.25$ & 2 & 4.35 & 7 \\
\hline Luminosity $\left(L_{\odot}\right)$ & $L$ & 25,000 & 3 & 14,000 & 7 \\
\hline Effective Temperature (K) & $T_{\text {eff }}$ & $48,000 \mathrm{~K}$ & 3 & 46,500 & 7 \\
\hline Magnitudes & $U, B, V$ & $8.57,9.73,10.01$ & 4 & $6.76,8.02,8.29$ & 8 \\
\hline Distance $(\mathrm{kpc})$ & $d$ & $2_{-0.6}^{+0.9}$ & 2 & $0.65 \pm 0.1$ & 6 \\
\hline Terminal wind velocity $\left(\mathrm{km} \mathrm{s}^{-1}\right)$ & $v_{\infty}$ & 2,000 & 3 & 1,350 & 9 \\
\hline Mass-loss rate $\left(M_{\odot} \mathrm{yr}^{-1}\right)$ & $\dot{M}$ & $10^{-8.5}$ & 3 & $10^{-8.5}$ & 7 \\
\hline
\end{tabular}

References: 1 - Husfeld 1987: 2 - Bauer \& Husfeld 1995: 3 - Jefferv \& Hamann 2010: 4 - Landolt 1973; 5 - Mereghetti et al. 2009; 6 - Kudritzki \& Simon 1978; 7 - Hamann 2010; 8 - Landolt \& Uomoto 2007; 9 - Hamann et al. 1981

\section{OBSERVATIONS AND DATA ANALYSIS}

$\mathrm{BD}+37^{\circ} 442$ was observed with $X M M-N e w t o n$ on 2011 August 21, starting at 23:35:19 UT (MJD = 55794.983). The three EPIC cameras, i.e. one $p n$ (Strüder et al. 2001) and two MOS (Turner et al. 2001), were operated in full frame mode, with time resolution of $73 \mathrm{~ms}$ for the $p n$ and of $2.6 \mathrm{~s}$ for the two MOS cameras; the effective source exposure time was, respectively, of $\sim 33 \mathrm{ks}$ and $\sim 28 \mathrm{ks}$. For all cameras the medium thickness filter was used.

We used version 11.0 of the XMM-Newton Science Analysis System $(S A S)$ to process the event files. The observation was affected by high instrumental background only for a short time interval of $\sim 1.2 \mathrm{ks}$, which we removed for the spectral analysis. We selected only the events with pattern in the range $0-4$ (i.e. mono- and bipixel events) for the $p n$ camera and 0-12 (i.e. from 1 to 4 pixel events) for the two MOS.

The images obtained with the three cameras clearly show that $\mathrm{BD}+37^{\circ} 442$ is emitting in the $\mathrm{X}$-ray range: in fact a source is significantly detected at the coordinates R.A. $=01^{h} 58^{m} 33.4^{s}$, Dec. $=+38^{\circ} 34^{\prime} 22.0^{\prime \prime}$, which differ by only $1.8^{\prime \prime}$ from the position of $\mathrm{BD}+37^{\circ} 442$. This difference is consistent with the $\sim 2^{\prime \prime}$ r.m.s. astrometric accuracy of $X M M-N$ ewton $\square$. The source net count rate in the $0.15-2 \mathrm{keV}$ range is $(1.62 \pm 0.08) \times 10^{-2}$ cts $\mathrm{s}^{-1}$ and $(1.9 \pm 0.2) \times 10^{-3}$ cts s$^{-1}$ for the $p n$ and each of the two MOS, respectively. $\mathrm{BD}+37^{\circ} 442$ is not detected above $\simeq 2 \mathrm{keV}$, suggesting a soft spectrum, as confirmed by the spectral analysis presented below.

For the timing analysis, we used the data of the whole observation and the three EPIC cameras, extracting the events from a circular region with radius $15^{\prime \prime}$ centered at the source position. We converted the arrival times to the solar system barycenter, then we combined the three datasets in a single event list. The backgroundsubtracted light curve of $\mathrm{BD}+37^{\circ} 442$ does not show variability on time scales from hundreds of seconds to the observation length. In order to look for periodicities, we examined 65,232 statistically independent periods in the range $1-10,000 \mathrm{~s}$ applying the phase-distribution test (Buccheri et al. 1983) to the counts in the $0.15-2$ $\mathrm{keV}$ energy range (685 counts). The resulting distribution of $Z^{2}$ statistics versus trial period is shown in

1
0018.ps.gz http://xmm2.esac.esa.int/docs/documents/CAL-TN-

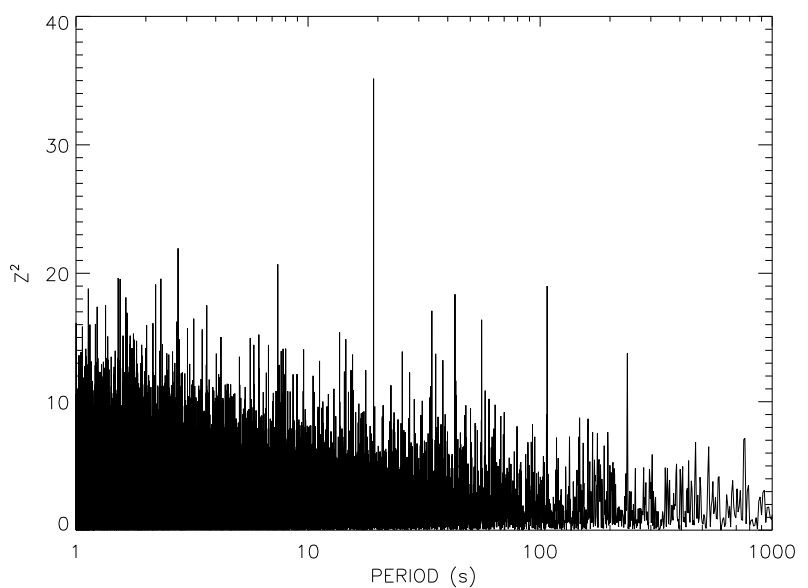

FIG. 1. Distribution of the $Z^{2}$ statistics versus trial period for the $\mathrm{BD}+37^{\circ} 442$ events in the $0.15-2 \mathrm{keV}$ energy range.

Fig [1] A peak value of $Z^{2}=35.15$ is found at the period $P=19.16 \mathrm{~s}$. The corresponding probability of chance occurrence, after taking into account the number of trials, is $1.5 \times 10^{-3}$. As shown by the folded and background-subtracted light curves of Fig. 2, the periodicity is visible also in the separate data sets, i.e. $p n$ and MOS1+MOS2, which give consistent phase distributions and pulsed fractions. The refined period value, obtained through pulse phase fitting, is $P=19.156 \pm$ $0.001 \mathrm{~s}$. The pulse profile shows a single broad peak with a $0.15-2 \mathrm{keV}$ pulsed fraction of $31 \pm 4 \%$ (amplitude of a sinusoid fitted to the folded light curve divided by the average flux). No significant differences are seen in the light curve shape and pulsed fraction in different energy ranges. We also performed a phase-resolved spectral analysis, as described below, for pairs of spectra corresponding to the pulse maximum/minimum or rise/decrease phase intervals, without finding significant differences in the spectral parameters.

Due to the limited signal-to-noise ratio of the MOS data, for the spectral analysis we considered only the $p n$ events. We generated the applicable response matrices and ancillary files using the $S A S$ tasks rmfgen and arfgen. To ensure the applicability of the $\chi^{2}$ statistics, the spectrum was rebinned with a minimum of 30 counts per bin and fitted using XSPEC (V 12.7.0). We only used the energy range $0.2-1 \mathrm{keV}$ since above $1 \mathrm{keV}$ the back- 

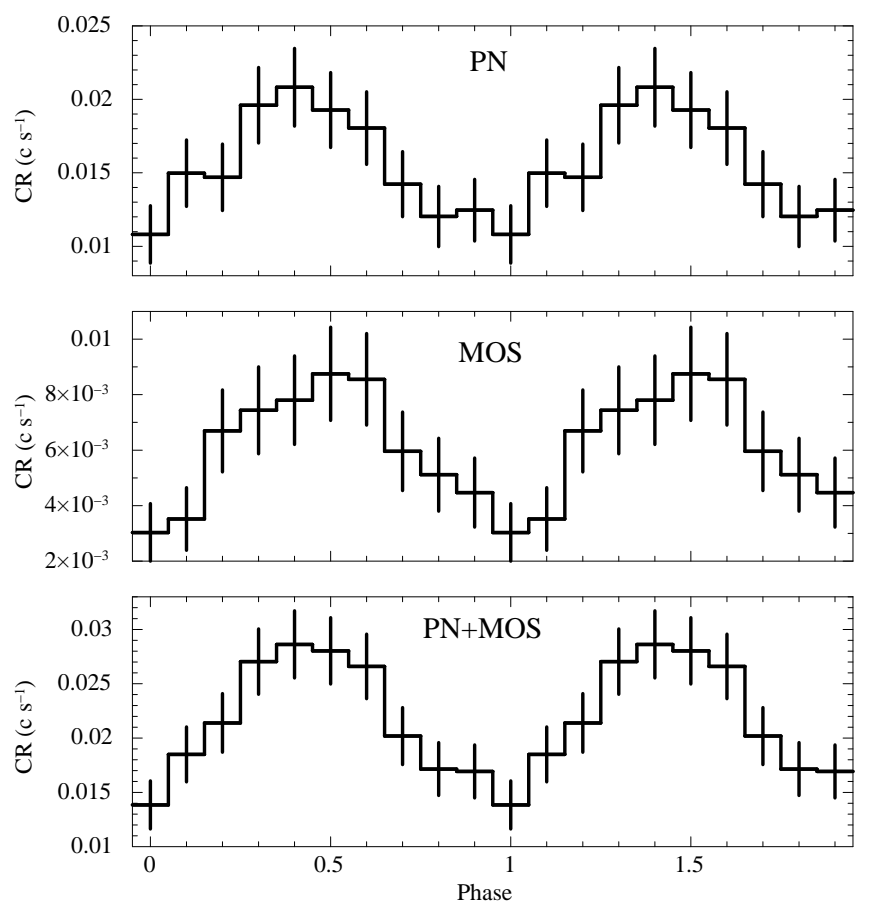

FIG. 2.- Background-subtracted light curves of BD $+37^{\circ} 442$ in the energy range $0.15-2 \mathrm{keV}$, folded at the best-fit period $P=$ 19.156 s. From top to bottom: pn data, sum of MOS1 and MOS2 data, total data (pn+MOS1+MOS2).

ground dominates and the source flux is negligible. In the following, all the spectral uncertainties and upper limits are given at the $90 \%$ confidence level for one interesting parameter, and we assume a source distance of $2 \mathrm{kpc}$ (Husfeld 1987).

The source spectrum is very soft: a fit with an absorbed power law gives an unphysically large photon index $(\Gamma \sim 5)$, while a blackbody gives a temperature $k T_{\mathrm{BB}}=93 \mathrm{eV}$. However, these single-component models give unacceptable fits $\left(\chi_{\nu}^{2}>2\right)$. A model consisting of an absorbed power law plus blackbody provides a significant improvement $\left(\chi_{\nu}^{2}=1.24\right.$ for 9 degrees of freedom, Fig 3), although the power-law photon index $\Gamma$ is poorly constrained in the range between -1 and 4 . Keeping $\Gamma$ fixed at its best fit value (2.25), the values of the other spectral parameters are: column density $N_{\mathrm{H}}=\left(1.4_{-0.6}^{+0.7}\right) \times 10^{21} \mathrm{~cm}^{-2}$, blackbody temperature $k T_{\mathrm{BB}}=45_{-9}^{+11} \mathrm{eV}$, and emitting radius $R_{\mathrm{BB}}=39_{-28}^{+162}$ $\mathrm{km}$. The absorbed flux in the energy range $0.2-1 \mathrm{keV}$ is $f_{\text {abs }, \mathrm{X}}=(2.6 \pm 0.3) \times 10^{-14} \mathrm{erg} \mathrm{cm}^{-2} \mathrm{~s}^{-1}$, of which $28 \%$ can be ascribed to the power-law component. The bolometric flux of the blackbody component, corrected for the absorption, is $1.7 \times 10^{-12} \mathrm{erg} \mathrm{cm}^{-2} \mathrm{~s}^{-1}$, corresponding to a luminosity of $7.8 \times 10^{32} \mathrm{erg} \mathrm{s}^{-1}$ (for a source distance $d=2 \mathrm{kpc}$ ). This value is subject to a large uncertainty because the value of $k T_{\mathrm{BB}}$ is only poorly constrained and depends on the slope of the power-law component. Therefore, in order to assess in a conservative way the uncertainty on the source luminosity, we repeated the spectral fits fixing the photon index at the values $\Gamma=1$ and $\Gamma=3$. In this way, we found acceptable values of $k T_{\mathrm{BB}}$ in the range $30-68 \mathrm{eV}$ (90\% c.l.), corresponding to bolometric blackbody luminosities between $6 \times 10^{31}$ and $1.5 \times 10^{35} \mathrm{erg} \mathrm{s}^{-1}$ (where the highest luminosity is obtained for the lowest temperature). The $0.2-10 \mathrm{keV}$ luminosity of the power-law component is in the range $(1.8-3.2) \times 10^{31} \mathrm{erg} \mathrm{s}^{-1}$.

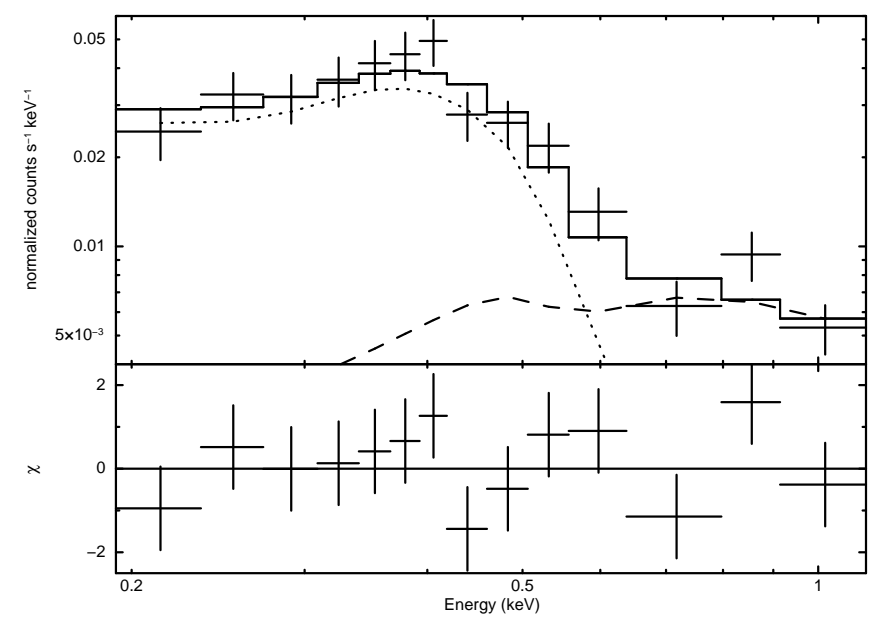

FIG. 3.- Top panel: pn spectrum of $\mathrm{BD}+37^{\circ} 442$ with the bestfit power-law (dashed line) plus blackbody (dotted line) model. Bottom panel: residuals (in units of $\sigma$ ) between data and model.

\section{DISCUSSION}

Our XMM-Newton observation of $\mathrm{BD}+37^{\circ} 442$ has provided the first detection of $\mathrm{X}$-ray emission from this O-type subdwarf. Shocks in the radiation-driven stellar winds of main sequence, giant, and supergiant early type stars give rise to soft $\mathrm{X}$-rays, with a typical ratio of $\mathrm{X}$-ray to optical flux of the order of $\log \left(f_{0.5-10 \mathrm{keV}}^{\text {unabs }} / f_{\mathrm{bol}}\right)=-6.45$ \pm 0.51 (Nazé 2009). It is not known if this relation holds also for low luminosity stars. The only other sdO star possibly detected in $\mathrm{X}$-rays is HD 49798, which is in a binary system with an $\mathrm{X}$-ray emitting white dwarf. In this system, the $\mathrm{X}$-ray flux of $f_{\mathrm{X}}=4.3 \times 10^{-14} \mathrm{erg} \mathrm{cm}^{-2} \mathrm{~s}^{-1}$, visible when the accreting WD companion is in eclipse, corresponds to $\log \left(f_{0.5-10 \mathrm{keV}}^{\text {unabs }} / f_{\mathrm{bol}}\right)=-7.4$ and could be due to emission from the sdO (Mereghetti et al. 2011b). For $\mathrm{BD}+37^{\circ} 442$ we measure $\log \left(f_{0.5-10 \mathrm{keV}}^{\text {unabs }} / f_{\mathrm{bol}}\right)=-$ 6.63 , consistent with the above values, but the significant periodic modulation at $19.156 \mathrm{~s}$ clearly indicates that most of, or possibly all, the detected flux originates in a compact companion star, rather than in $\mathrm{BD}+37^{\circ} 442$ itself. The periodic modulation can be explained equally well as the spin period of a NS or of a WD.

Ultraviolet spectra of $\mathrm{BD}+37^{\circ} 442$, acquired with the IUE satellite, show $\mathrm{N} \mathrm{V}$ and $\mathrm{C}$ IV resonance lines with $\mathrm{P}$ Cygni-like profiles, indicating the presence of a stellar wind (Rossi et al. 1984). A more recent analysis of UV and optical spectra of $\mathrm{BD}+37^{\circ} 442$ has been reported by Jeffery \& Hamann (2010), who derived a mass loss rate of $3 \times 10^{-9} M_{\odot} \mathrm{yr}^{-1}$ and a wind terminal velocity of $v_{\infty}=2,000 \mathrm{~km} \mathrm{~s}^{-1}$. It is possible that part of the sdO stellar wind is captured by its compact companion, giving rise to accretion powered $\mathrm{X}$-ray emission. The density and velocity of the wind material at the position of the compact object can be computed assuming a canonical wind velocity law with radial dependence $v(R)=v_{\infty}(1-1 / R)^{\beta}$, where $R$ is the radial distance in units of stellar radii and the index $\beta$ is typically in the 
range 0.6-1. Assuming for simplicity Bondi-Hoyle accretion, and relating the orbital separation to the period of a circular orbit $P_{\text {orb }}$ through Kepler's law, we can derive the expected accretion luminosity as a function of $P_{\text {orb }}$ shown in Fig. 4. The curves refer to a NS of $1.4 M_{\odot}$ and $10 \mathrm{~km}$ radius, and to a WD of $0.6 M_{\odot}$ and $10,000 \mathrm{~km}$ radius; in both cases we used for $\mathrm{BD}+37^{\circ} 442$ a mass of $0.9 M_{\odot}$ (Husfeld 1987). We have also indicated in the figure the allowed range of luminosity of the blackbody component (for simplicity we do not consider the additional $30 \%$ contribution to the total luminosity coming from the power-law component).

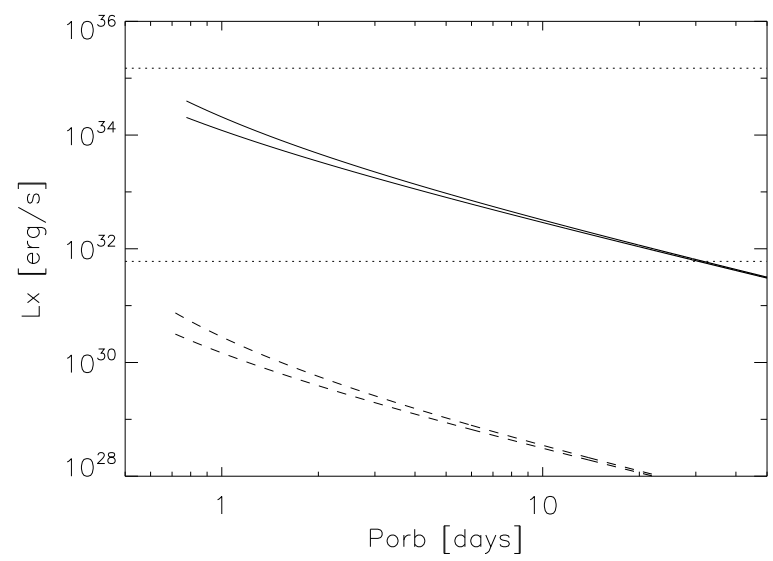

FIG. 4.- Estimated wind-accretion X-ray luminosity of $\mathrm{BD}+37^{\circ} 442$ as a function of the binary period, in the case of a WD (dashed lines) and of a NS (solid lines) The couples of lines refer to two values of the wind velocity index $\beta=0.6$ and $\beta=1$. The horizontal dotted lines represent the minimum and maximum of the estimated luminosity of the blackbody component.

Although the observed luminosity is only poorly constrained due to the large uncertainties in the spectral parameters, it is clearly consistent with that expected from a $\mathrm{NS}$ orbiting $\mathrm{BD}+37^{\circ} 442$ with a period between about one and several days. The blackbody emitting radius derived from the best fit, $R_{\mathrm{BB}}=39_{-28}^{+162} \mathrm{~km}$ (for $d=$ $2 \mathrm{kpc}$ ), is only marginally consistent with a neutron star. However, this parameter is strongly correlated with the poorly constrained slope of the power law spectral component and an acceptable fit can be obtained, e.g., with $\Gamma=1.5, k T_{\mathrm{BB}} \sim 58 \mathrm{eV}$, and $R_{\mathrm{BB}} \sim 10 \mathrm{~km}$. Alternatively, the accreting companion could be a WD, if the sdO extends to (or close to) the Roche-lobe, thus yielding an accretion rate larger than what we computed assuming wind accretion (or in the unlikely case that the adopted distance is largely overestimated). The observed spectrum is much softer than the typical spectrum of neutron stars in classical X-ray binaries, which however have very different companion stars and accrete at higher rates. It should also be considered that we can observe the low temperature emission from BD $+37^{\circ} 442$ (and HD 49798, see below) only because these sources are at high Galactic latitude, where interstellar X-ray absorption is very small.

Up to now $\mathrm{BD}+37^{\circ} 442$ was believed to be a single star: no evidence for a companion was seen in spectroscopic (Fä̈ et al. 1973; Kaufmann \& Theil 1980; Dworetsky et al. 1982) or photometric data (Landolt 1968, 1973), and infrared observations did not show any excess emission (Theill et al. 1995). On the other hand, some of the very few published radial velocity measurements are inconsistent with a single value, hinting to a possible binary motion. A radial velocity $V_{\mathrm{r}}=-156.4$ $\pm 1.1 \mathrm{~km} \mathrm{~s}^{-1}$ is given in the original discovery paper (Rebeirot 1966), while Drilling \& Heber (1987) found $V_{\mathrm{r}}$ $=-94 \pm 1 \mathrm{~km} \mathrm{~s}^{-1}$. New accurate radial measurements are needed to determine the parameters of this binary.

The $\mathrm{X}$-ray properties of $\mathrm{BD}+37^{\circ} 442$ are very similar to those of the only other $\mathrm{X}$-ray source associated with an sdO star: HD 49798. In this binary a massive white dwarf accretes matter from the stellar wind of its subdwarf companion (Mereghetti et al. 2009). Also in the case of HD 49798 the X-ray emission is pulsed, with a period $(13.2 \mathrm{~s})$ similar to that we have discovered in $\mathrm{BD}+37^{\circ} 442$, and the soft spectrum is well described by the sum of a blackbody with $k T_{\mathrm{BB}}=39 \mathrm{eV}$ and a power law with $\Gamma=1.6$ (Mereghetti et al. 2011b). HD 49798 has an orbital period of 1.55 days, first discovered through radial velocity measurements of the sdO optical emission (Thackeray 1970), and an X-ray luminosity of $10^{32} \mathrm{erg} \mathrm{s}^{-1}$ (at $d=650 \mathrm{pc}$ ).

The fact that the only two sdO stars observed with sensitive $\mathrm{X}$-ray instruments turned out to be binaries with a compact companion is noteworthy. Although it is premature to draw conclusions from such small numbers, this might suggest that the fraction of sdO binaries is larger than currently believed on the basis of optical observations. Note that, contrary to late type companions, which can give a detectable contribution in the spectral and photometric data, WDs and NSs are too faint and completely outshined in the optical/UV by the sdO emission. Both $\mathrm{BD}+37^{\circ} 442$ and HD 49798 belong to the subclass of luminous, He-rich sdOs, whose origin and evolutionary link with other classes of stars is still unclear (Napiwotzki 2008; Justham et al. 2011). In this respect, $\mathrm{X}$-ray observations might provide important information to complement optical/UV data. Besides the potential of discovering other sdO binaries through the detection of pulsations, the high sensitivity of current satellites like $X M M-N e w t o n$ and Chandra can probe the $\mathrm{X}$-ray emission of single sdOs, and test whether their wind emission scales as in early type stars of higher luminosity.

We wish to thank U. Heber and S. Geier for useful discussions. This work is based on observations obtained with $X M M-N e w t o n$, an ESA science mission with instruments and contributions directly funded by ESA Member States and NASA. We acknowledge financial contributions by the Italian Space Agency through ASI/INAF agreements I/009/10/0 and I/032/10/0 for, respectively, the data analysis and the $X M M-N$ ewton operations. P.E. acknowledges financial support from the Autonomous Region of Sardinia through a research grant under the program PO Sardegna FSE 2007-2013, L.R. $7 / 2007$ 'Promoting scientific research and innovation technology in Sardinia'. 


\section{REFERENCES}

Bauer, F. \& Husfeld, D, 1995, A\&A, 300, 481

Buccheri, R., Bennett, K., Bignami, G. F., et al. 1983, A\&A, 128, 245

Copperwheat, C. M., Morales-Rueda, L., Marsh, T. R., Maxted, P. F. L., \& Heber, U. 2011, MNRAS, 415, 1381

Drilling, J. S. \& Heber, U. 1987, in IAU Colloq. 95: Second Conference on Faint Blue Stars, ed. A. G. D. Philip, D. S. Hayes, \& J. W. Liebert, 603-606

Dworetsky, M. M., Whitelock, P. A., \& Carnochan, D. J. 1982, MNRAS, 201, 901

Edelmann, H., Heber, U., Altmann, M., Karl, C., \& Lisker, T. 2005, A\&A, 442, 1023

Faÿ, T., Honeycutt, R. K., \& Warren, Jr., W. H. 1973, AJ, 78, 246

Geier, S., Heber, U., Podsiadlowski, P., et al. 2010, A\&A, 519, A25

Hamann, W.-R. 2010, Ap\&SS, 329, 151

Hamann, W.-R., Gruschinske, J., Kudritzki, R. P., \& Simon, K. P. 1981, A\&A, 104, 249

Han, Z., Podsiadlowski, P., Maxted, P. F. L., Marsh, T. R., \& Ivanova, N. 2002, MNRAS, 336, 449

Heber, U. 2009, ARA\&A, 47, 211

Heber, U., Hirsch, H., Ströer, A., et al. 2006, Baltic Astronomy, 15,91

Heber, U. \& Jeffery, C. S., eds. 1992, Lecture Notes in Physics, Berlin Springer Verlag, Vol. 401, The Atmospheres of Early-Type Stars

Hirsch, H. A., Heber, U., \& O'Toole, S. J. 2008, in Astronomical Society of the Pacific Conference Series, Vol. 392, Hot Subdwarf Stars and Related Objects, ed. U. Heber, C. S. Jeffery, \& R. Napiwotzki, 131

Husfeld, D. 1987, in IAU Colloq. 95: Second Conference on Faint Blue Stars, ed. A. G. D. Philip, D. S. Hayes, \& J. W. Liebert, 237-246

Israel, G. L., Stella, L., Angelini, L., et al. 1997, ApJ, 474, L53
Jeffery, C. S. \& Hamann, W.-R. 2010, MNRAS, 404, 1698

Justham, S., Podsiadlowski, P., \& Han, Z. 2011, MNRAS, 410, 984

Kaufmann, J. P. \& Theil, U. 1980, A\&AS, 41, 271

Koen, C., Orosz, J. A., \& Wade, R. A. 1998, MNRAS, 300, 695

Kudritzki, R. P. \& Simon, K. P. 1978, A\&A, 70, 653

Landolt, A. U. 1968, PASP, 80, 318

-. 1973, PASP, 85, 661

Landolt, A. U. \& Uomoto, A. K. 2007, AJ, 133, 768

Maxted, P. F. L., Heber, U., Marsh, T. R., \& North, R. C. 2001 , MNRAS, 326, 1391

Maxted, P. F. L., Marsh, T. R., \& North, R. C. 2000, MNRAS, 317, L41

Mereghetti, S., Campana, S., Esposito, P., La Palombara, N., \& Tiengo, A. 2011a, A\&A, 536, A69

Mereghetti, S., La Palombara, N., Tiengo, A., et al. 2011b, ApJ, 737,51

Mereghetti, S., Tiengo, A., Esposito, P., et al. 2009, Science, 325, 1222

Napiwotzki, R. 2008, in Astronomical Society of the Pacific Conference Series, Vol. 392, Hot Subdwarf Stars and Related Objects, ed. U. Heber, C. S. Jeffery, \& R. Napiwotzki, 139 Napiwotzki, R., Karl, C. A., Lisker, T., et al. 2004, Ap\&SS, 291, 321

Nazé, Y. 2009, A\&A, 506, 1055

Rebeirot, E. 1966, C.r.hebd. Seanc. Acad. Sci. Paris, 262, 1105

Rossi, L., Viotti, R., \& Altamore, A. 1984, A\&AS, 55, 361 Strüder, L., Briel, U., Dennerl, K., et al. 2001, A\&A, 365, L18 Thackeray, A. D. 1970, MNRAS, 150, 215

Thejll, P., Ulla, A., \& MacDonald, J. 1995, A\&A, 303, 773

Turner, M. J. L., Abbey, A., Arnaud, M., et al. 2001, A\&A, 365, L27 